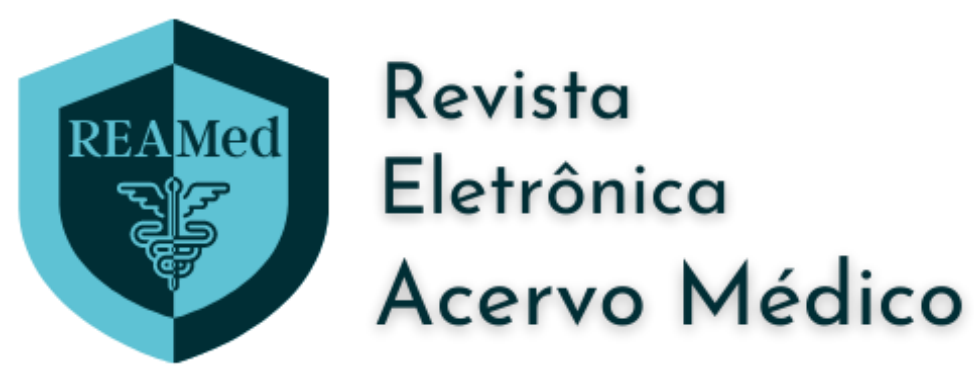

\title{
Efeitos dos transtornos de ansiedade nas doenças cardiovasculares: uma revisão integrativa
}

\author{
Effects of anxiety disorders on cardiovascular disease: an integrative review \\ Efectos de los trastornos de ansiedad en las enfermedades cardiovasculares: una \\ revisión integradora
}

Suzana Tomaz Vasconcelos ${ }^{1 *}$, Amanda Prete Barros ${ }^{2}$, Amanda Santos Rodrigues ${ }^{3}$, Gianne Silva Vetorasso $^{2}$, João Pedro Murcia Sanchez ${ }^{2}$, Luana Silva Pinheiro ${ }^{3}$, Mônica Maria Araújo Sousa ${ }^{1}$, Nicolle Tomás Arcanjo Vasconcelos ${ }^{3}$, Rady Martins de Almeida ${ }^{1}$ e Ariany de Assis Moura ${ }^{1}$.

\section{RESUMO}

Objetivo: Analisar a relação entre ansiedade e Doença Cardiovascular (DCV), pontuar os melhores métodos diagnósticos e sintetizar as terapias da ansiedade em pacientes com comorbidades cardíacas. Métodos: Trata-se de uma revisão integrativa que utilizou as bases de dados Scielo e Pubmed para busca dos descritores: "Anxiety Disorders", "Cardiovascular disease", "Hypertension" e "Comorbidity". Encontrou-se 406 artigos e selecionou-se 15 para compor essa revisão. Resultados: DCV é uma das principais causas de morbimortalidade e tem a ansiedade como uma de seus fatores de risco. Sabe-se que pacientes com algum transtorno ansioso e DCV têm maiores taxas de mortalidade, não adesão terapêutica e não engajamento a estilos saudáveis. é recomendado um diagnóstico precoce e rastreamento de rotina dessa doença em pacientes cardíacos através de manuais e diretrizes e como terapia farmacológica sugere os antidepressivos Inibidores Seletivos da Recaptação de Serotonina e os benzodiazepínicos. Ainda, indica a psicoterapia e atividade física para os transtornos de ansiedade em pacientes com DCV. Conclusão: Sabe-se que ansiedade piora o prognóstico de das DCVs, mas não apresenta relações bem esclarecidas com essas patologias. Por isso, necessita de mais estudos para esclarecer esses mecanismos e reafirmar a necessidade de rastreamento mental nos pacientes com agressão cardíaca.

Palavras-chave: Transtorno de ansiedade, Doença cardiovascular, Diagnóstico e tratamento.

\begin{abstract}
Objective: Analyze the relation between anxiety and cardiovascular disease (CVD), point the best diagnostic methods and synthesize anxiety therapies in patients with cardiac comorbidities. Methods: This is an integrative review that used the Scielo and Pubmed databases to search for the descriptors: "Anxiety Disorders", "Cardiovascular disease", "Hypertension" and "Comorbidity". 406 articles were found and 15 were
\end{abstract}

\footnotetext{
1 Universidade de Fortaleza (UNIFOR), Fortaleza - CE. *E-mail: suzy_tomaz_@hotmail.com

${ }^{2}$ Centro Universitário de Votuporanga (UNIFEV), Votuporanga - SP.

${ }^{3}$ Centro Universitário Christus (UNICHRISTUS), Fortaleza - CE.
} 
selected for this review. Results: CVD is the main cause of morbidity/mortality, being anxiety one of it's risk factors. Patients affected with both anxiety disorder and CVD have higher mortality rates, poor therapeutic adherence and a more difficult commitment to a healthy lifestyle. Therefore, it's recommended an early diagnosis and routine screening of this disease in cardiac patients through manuals and guidelines. Regarding pharmacological therapy, the selective serotonin reuptake inhibitor and benzodiazepines are the main choices. Psychotherapy and physical activity must be associated with pharmacological therapy for better therapeutic results. Final considerations: Anxiety worsens the prognosis of CVD's, but it doesn't have a clear relation with these pathologies. Therefore, further studies are needed to clarify these mechanisms and reaffirm the need for mental tracking in patients with cardiac injury.

Key words: Anxiety disorders, Cardiovascular disease, Diagnoses and treatment.

\section{RESUMEN}

Objetivo: Analizar la relación entre ansiedad y la Enfermedade Cardiovascular (ECV), puntuar los mejores métodos diagnósticos y sintetizar terapias de ansiedad en pacientes cardíacos. Métodos: Revisión integradora que utilizó las bases de datos Scielo y Pubmed para buscar los descriptores: "Anxiety Disorders", "Cardiovascular disease", "Hypertension" y "Comorbidity". Encontró 406 artículos y seleccionó 15 para componer esta revisión. Resultados: ECV es una de las principales causas de morbilidad y mortalidad y la ansiedad es uno de sus factores de riesgo. Se sabe que los pacientes con algún trastorno de ansiedad y ECV presentan mayores tasas de mortalidad, no adherencia terapéutica y no compromiso con estilos saludables. Se recomienda un diagnóstico precoz y cribado de rutina de esta enfermedad en pacientes cardíacos mediante manuales y guías y como la terapia farmacológica sugiere los antidepresivos inhibidores selectivos de la recaptación de serotonina y benzodiazepinas. También indica psicoterapia y actividad física para los trastornos de ansiedad en pacientes con ECV. Consideraciones finales: Se sabe que la ansiedad empeora el pronóstico de las ECV, pero no tiene una relación clara con estas patologías. Por lo tanto, se necesitan más estudios para aclarar estos mecanismos y reafirmar la necesidad de seguimiento mental en pacientes con lesión cardíaca.

Palabras clave: Trastorno de ansiedad, Enfermedad cardiovascular, Diagnóstico y tratamiento.

\section{INTRODUÇÃO}

Os Transtornos de Ansiedade são considerados como um grande fardo para a saúde pública. Estima-se que essas condições são as mais prevalentes dentre todas as doenças mentais. De acordo com alguns estudos, espera-se que um terço de toda a população global seja acometida por algum desses transtornos, sendo considerado como o sexto colaborador para a deficiência. O grupo de doenças que fazem parte desse desarranjo é o Transtorno de Ansiedade Generalizada (TAG), Transtorno do Pânico (TP), fobias, Transtorno Obsessivo-Compulsivo (TOC) e Transtorno de Estresse Pós-Traumático (TSPT) (JOHNSON HM, 2019).

As DCVs, sendo doenças do coração e dos vasos sanguíneos, são consideradas como uma das principais causas de mortalidade e uma das patologias mais prevalentes. Diversos estudos reconhecem a ansiedade como fator de risco do mesmo nível daqueles já consagrados pela literatura, como o tabagismo, sedentarismo e dieta rica em gorduras. Assim, essa doença somática pode explicar, em parte, a grande morbimortalidade desses distúrbios cardíacos (GARCÍA-BATISTA ZE, et al., 2020).

Estudos recentes apontam para altas chances de a ansiedade aumentar o risco de desenvolvimento das Doenças Cardiovasculares (DCVs). No entanto, é difícil definir com precisão uma relação causal. Ainda, devido ao fato de ter uma sobreposição entre os sintomas psicológicos e as DCVs há uma dificuldade diagnóstica dessas doenças somáticas nos pacientes com distúrbios cardíacos. Dentre o grupo de patologias que compõe as DCV, pode-se destacar como as mais importantes a Hipertensão Arterial Sistêmica (HAS), o Infarto Agudo do Miocárdio (IAM), a Doença Arterial Coronariana (DAC) e a Insuficiência Cardíaca (IC) (ANGERMANN CE e ERTL G, 2018; KANG H, et al., 2017; TULLY PJ, et al., 2016). 
Teorias biológicas e comportamentais tem sido proposta para explicar os mecanismos envolvidos entre a ansiedade e as DCVs. Uma das mais bem aceitas é que essa desordem psicossomática implica em uma atividade disfuncional do Sistema Nervoso Autonômico (SNA) e do eixo hipotálamo-hipófise-adrenal, afetando, assim, o sistema cardiovascular. Porém, sabe-se que nem sempre as doenças aparecem nessa ordem, ou seja, primeiro ansiedade e logo depois alguma DCV, uma vez que após o paciente descobrir que tem alguma comorbidade cardíaca, isso pode gerar medos, anseios e frustrações, acarretando em quadros ansiosos. De qualquer forma, é inegável a influência negativa da ansiedade para o coração, sendo essa relação agravada quando há uma associação com outros quadros psicológicos, como a depressão (REAVELL J, et al., 2018).

Apesar da ansiedade já ter evidências como um dos grandes riscos para as DCVs, é importante que essa doença não seja vista apenas como um fator de risco, uma vez que ela pode começar mesmo em pacientes que já tinham uma DCV estabelecida. Isso se torna necessário uma vez que ela tem se mostrado também como um fator preditivo para uma baixa adesão à reabilitação cardíaca e à terapia farmacológica. Assim, alguns estudos afirmam que a ansiedade está associada a comportamentos não saudáveis, como o tabagismo, o sedentarismo e o consumo excessivo de álcool, piorando o prognóstico do paciente (CELANO CM, et al., 2018; GARCÍA-BATISTA ZE, et al., 2020; MACHADO MB, et al., 2016).

Ainda, a ansiedade aumenta a probabilidade de eventos adversos em pacientes com alguma DCV já estabelecida, como insuficiência ventricular esquerda, acidente vascular encefálico. No entanto, os efeitos prognósticos dessa doença dependem de cada subtipo da ansiedade já mencionados, fazendo com que seja necessário não apenas o diagnóstico de ansiedade, mas também a distinção entre os vários tipos de transtornos (TULLY PJ, et al., 2016).

Para fazer o diagnóstico de ansiedade, a parte clínica é extremamente fundamental, apesar de muitos sintomas confundirem com doenças cardíacas e respiratórias. Existem diversas diretrizes e manuais que auxiliam o médico na hora de identificar essa patologia. Recomenda-se que sempre que possível seja feito um rastreamento de rotina para essas doenças em pacientes com DCV, uma vez que são grandes influenciadoras nas altas taxas de morbimortalidade na doença cardíaca e ainda acarretam em uma baixa adesão terapêutica e de hábitos saudáveis de vida (ANGERMANN CE e ERTL G, 2018; TULLY PJ, et al., 2016).

Na terapia para ansiedade nos pacientes com DCV, é fortemente contraindicado o uso de antidepressivos tricíclicos, optando pelos antidepressivos Inibidores Seletivos da Recaptação de Serotonina (ISRS) e pelos benzodiazepínicos, a exemplo o Clonazepam e o Alprazolam. Ainda, os profissionais podem utilizar de terapias não medicamentosas, como a atividade física e a Terapia Cognitivo-Comportamental (TCC), pois, de acordo com vários estudos, elas são eficientes para diminuir a morbimortalidade causada pela ansiedade (ANGERMANN CE e ERTL G, 2018).

A identificação de novas estratégias para a prevenção e tratamento da ansiedade em pacientes com DCV estabelecida é de extrema importância no combate da morbimortalidade e na elaboração de melhores condições de vida. Diante do exposto, o presente estudo teve como objetivo analisar o que a literatura atual diz a respeito da relação entre ansiedade e DCV, bem como pontuar os melhores métodos para diagnóstico preciso dessa doença psicossomática e evidências terapêuticas dessas patologias na população com comorbidades cardíacas.

\section{MÉTODOS}

O trabalho consiste em uma Revisão Integrativa e, para a sua elaboração, realizou-se uma busca nas seguintes bases de dados: PubMed e Scientific Electronic Library Online (Scielo). Foram utilizados, de acordo com os Descritores em Ciências da Saúde (DeCS), "Anxiety Disorders", "Cardiovascular disease", "Hypertension" e "Comorbidity" em associação com os operadores booleanos "AND" e "OR", para fazer a busca dos artigos. A partir dessa pesquisa, foram encontrados 406 artigos e selecionados 15 artigos completos (Figura 1). 
Como filtros, foram usados artigos com resultados compreendidos no período de 2016 a 2020 e em todos os idiomas. Excluíram-se os artigos que não corroboram com o tema, não tinham veracidade ou não apresentavam dados relevantes para este estudo. Os critérios de inclusão compreendiam artigos que abordavam a relação entre a ansiedade e sua influência em doenças cardiovasculares.

Figura 1 - Resultado da busca bibliográfica.

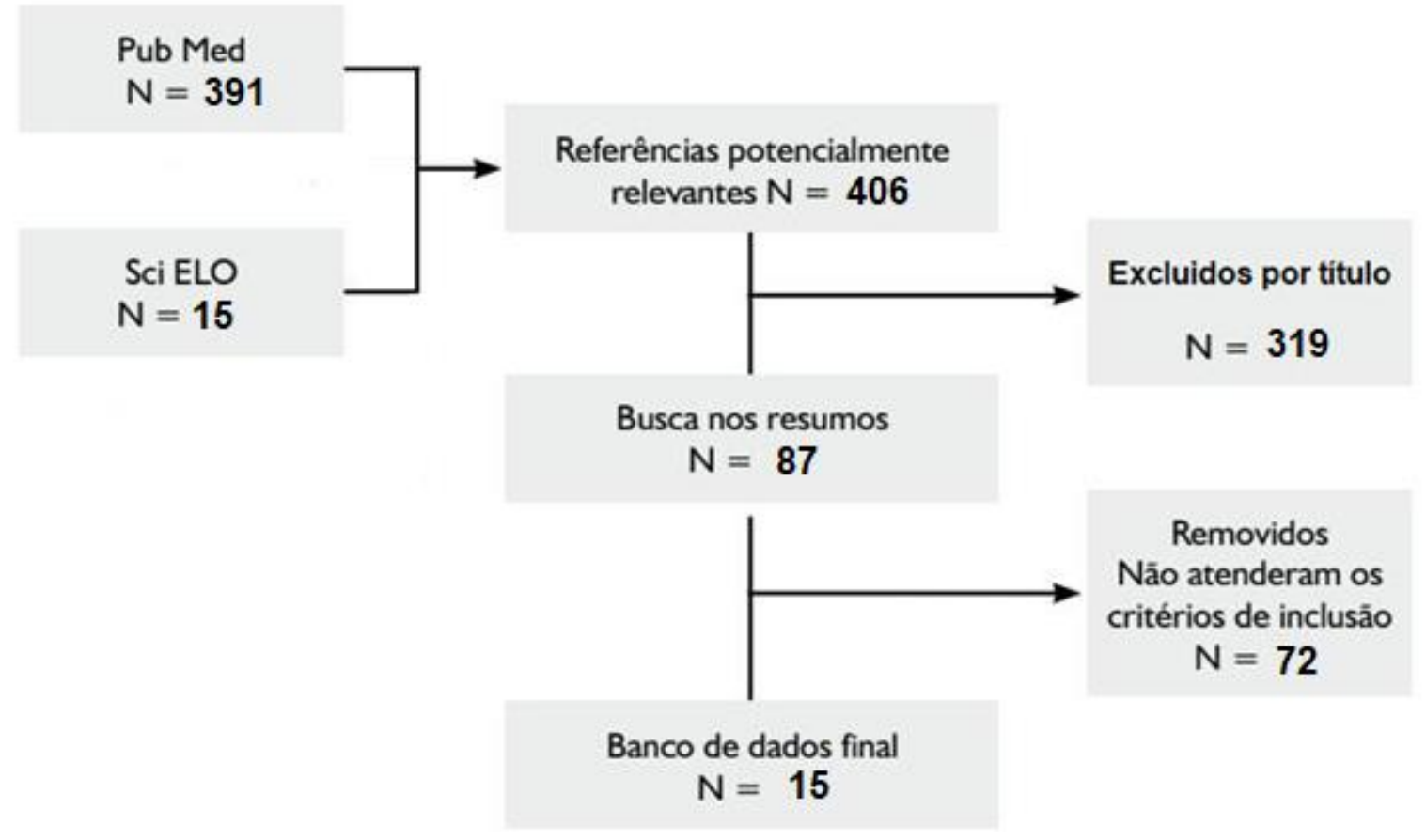

Fonte: Vasconcelos ST, et al., 2021.

\section{RESULTADOS}

Após a busca nas bases de dados sobre o tema ansiedade no risco cardiovascular, foram encontrados 406 artigos, selecionados os publicados entre 2016 e 2020 e utilizados 15 para compor esta revisão. Os artigos selecionados estão representados no Quadro 1. 
Quadro 1 - Síntese dos principais achados sobre ansiedade e DCV dentre os 15 artigos selecionados.

\begin{tabular}{|c|c|c|}
\hline $\mathbf{N}$ & Autores (Ano) & $\begin{array}{ll} & \text { Principais achados } \\
\end{array}$ \\
\hline 1 & ANGERMANN CE e ERTL G (2018) & $\begin{array}{l}\text { Esse estudo consiste em uma revisão de literatura e uma metanálise sobre o conhecimento atual da influência dos } \\
\text { transtornos mentais em DCV e insuficiência cardíaca. Como um dos pontos-chave abordados no artigo, têm-se as } \\
\text { interações bidirecionais entre o Sistema Nervoso Central e o cardiovascular. Em conclusão, o estudo afirma a } \\
\text { necessidade de cuidados de rotina para o rastreamento dessas doenças somáticas, bem como o tratamento } \\
\text { multidisciplinar com a participação ativa do paciente. }\end{array}$ \\
\hline 2 & CELANO CM, et al. (2018) & $\begin{array}{l}\text { Consiste em uma revisão de literatura com o objetivo de identificar as associações entre depressão, ansiedade e } \\
\text { insuficiência cardíaca, examinar os mecanismos entre essas condições e os resultados médicos, identificar os } \\
\text { melhores métodos para diagnóstico eficaz dessas doenças psicossomáticas e revisar as evidências atuais para o } \\
\text { tratamento dessas condições. Como resultados, obteve que os pacientes com Transtornos de Ansiedade e } \\
\text { Insuficiência Cardíaca apresentam baixa adesão ao tratamento, aumento de hospitalizações e mortalidade elevada. } \\
\text { Além disso, a entrevista clínica e o diagnóstico formal é o melhor processo de avaliação, assim como a terapia } \\
\text { cognitivo-comportamental e os inibidores seletivos da recaptação de serotonina são alternativas terapêuticas eficazes. }\end{array}$ \\
\hline 3 & FOLDES-BUSQUE G, et al. (2020) & $\begin{array}{l}\text { Trata-se de um estudo de coorte longitudinal que teve como objetivo estimar a prevalência e incidência do Transtorno } \\
\text { de Ansiedade Generalizada (TAG) e Transtorno do Pânico (TP) em pacientes com Doença Arterial Coronariana por } 2 \\
\text { anos e avaliar prospectivamente a associação entre TAG ou TP e eventos cardíacos adversos, adesão ao tratamento, } \\
\text { qualidade de vida e sofrimento psíquico. Como conclusão, afirma a necessidade de desenvolver recomendações } \\
\text { clínicas claras para o manejo dessas doenças, melhorando o prognóstico do paciente em ambas as condições. }\end{array}$ \\
\hline 4 & GARCÍA-BATISTA ZE, et al. (2020) & $\begin{array}{l}\text { Esse estudo tem um desenho de caso-controle com o objetivo de verificar os níveis de depressão, ansiedade e raiva } \\
\text { em pessoas que sofreram um ataque do miocárdio. Dividiu-se, então, a população em dois grupos: um com pessoas } \\
\text { que sofreram ataque do miocárdio e outro controle pareado por idade e sexo. Como resultado obteve que, embora } \\
\text { todas as variáveis apresentem níveis elevados no grupo de DCV, apenas a depressão tem diferença estatisticamente } \\
\text { significativa. Assim, como conclusão o estudo explica a presença de comorbidade depressiva em pessoas que } \\
\text { sofreram acidente miocárdico. }\end{array}$ \\
\hline 5 & HE C, et al. (2020) & $\begin{array}{l}\text { Consiste em um estudo de coorte prospectivo que teve como hipótese a associação da ansiedade com resultados } \\
\text { clínicos em pacientes com infarto do miocárdio na ausência de doença arterial coronariana obstrutiva. Como resultado, } \\
\text { obteve que os eventos cardiovasculares adversos e a mortalidade por todas as causas tiveram valores maiores no } \\
\text { grupo com ansiedade em comparação com o grupo sem ansiedade. Assim, o artigo conclui que a ansiedade está } \\
\text { significativa e independentemente associada com o aumento desses eventos nos pacientes estudados. }\end{array}$ \\
\hline
\end{tabular}




\begin{tabular}{|c|c|c|}
\hline $\mathbf{N}$ & Autores (Ano) & Principais achados \\
\hline 6 & JOHNSON HM (2019) & $\begin{array}{l}\text { Trata-se de uma revisão de literatura sobre a associação entre transtornos de ansiedade comórbidos e hipertensão. } \\
\text { Como descoberta, teve que a variabilidade da pressão arterial e a sensibilidade barorreflexa reduzida como feedback } \\
\text { à disfunção autonômica fornecem uma compreensão das relações/mecanismos entre ansiedade e hipertensão. } \\
\text { Conclui-se, então, uma associação positiva entre essa doença somática e a hipertensão, além de hipóteses } \\
\text { mecanicistas para essa relação. }\end{array}$ \\
\hline 7 & KANG H, et al. (2017) & $\begin{array}{l}\text { Esse estudo de coorte prospectivo teve como objetivo investigar os efeitos longitudinais da ansiedade, depressão e } \\
\text { suas comorbidades em distúrbios físicos e incapacidade em população idosa. Como resultado, obteve que a ansiedade } \\
\text { por si só foi associada à incidência de doenças cardíacas. Além disso, ansiedade e depressão comórbidas foram } \\
\text { associadas a um aumento do número de distúrbios físicos e no grau de incapacidade no seguimento de dois anos do } \\
\text { estudo. Concluiu-se que a ansiedade e depressão devem ser avaliadas na população idosa e a necessidade de } \\
\text { estudos futuros para determinar as intervenções nessas doenças e minimizar seus impactos. }\end{array}$ \\
\hline 8 & LI J, et al. (2020) & $\begin{array}{l}\text { Trata-se de uma metanálise que teve como objetivo avaliar a associação entre ansiedade e resultados clínicos em } \\
\text { pacientes com Síndrome Coronariana Aguda (SCA) e investigar o papel potencial da depressão nessa associação. } \\
\text { Como resultado, teve que a ansiedade foi independentemente associada ao aumento do risco de mortalidade (RR } \\
1,21 ; \text { IC } 95 \% 1,07 \text { a 1,37). Além disso, a ansiedade não foi significativamente associada ao risco de mortalidade após } \\
\text { o ajuste para depressão. Como conclusão, teve uma associação positiva entre ansiedade e SCA, mas uma parte } \\
\text { dessa associação pode ser confundida por sintomas depressivos simultâneos. }\end{array}$ \\
\hline 9 & LI P, et al. (2020) & $\begin{array}{l}\text { Consiste em um estudo de coorte retrospectivo que teve como hipótese examinar os efeitos dos transtornos de } \\
\text { ansiedade concorrentes sobre os resultados hospitalares em pacientes com infarto do miocárdio. Nos resultados, } \\
\text { notou-se que o grupo com ansiedade teve uma incidência menor de mortalidade hospitalar ( } 3 \% \text { vs } 4 \% \text { ), choque } \\
\text { cardiogênico ( } 4,9 \% \text { vs } 5,6 \% \text { e arritmia ventricular }(6,7 \% \text { vs } 7,9 \%) \text { em relação ao grupo sem ansiedade. Porém, esses } \\
\text { valores significativos só foram notados no subgrupo sem supra do segmento ST. Assim, não houve melhores } \\
\text { resultados para o subgrupo com supra do segmento ST. }\end{array}$ \\
\hline 10 & MACHADO MB, et al. (2016) & $\begin{array}{l}\text { Esse estudo transversal com base em dados populacionais teve como objetivo avaliar a prevalência de transtornos } \\
\text { ansiosos e fatores associados em uma amostra populacional de idosos. Foi avaliado, ainda, a hipertensão, infarto } \\
\text { agudo do miocárdio e a dosagem de colesterol. Como resultado, teve que a maior prevalência foi do Transtorno de } \\
\text { Ansiedade Generalizada (TAG). Ainda, percebeu-se que a hipertensão era mais prevalente em indivíduos com TOC e } \\
\text { o infarto foi associado à fobia social. O estudo concluiu que quadros de ansiedade são frequentes na população idosa } \\
\text { e estão associados a algumas variáveis clínicas cardiovasculares. }\end{array}$ \\
\hline
\end{tabular}




\begin{tabular}{|c|c|c|}
\hline $\mathbf{N}$ & Autores (Ano) & Principais achados \\
\hline 11 & PELTZER S, et al. (2020) & $\begin{array}{l}\text { Trata-se de um estudo transversal que teve como objetivo mapear o estado de diagnóstico e tratamento de doenças } \\
\text { mentais em pacientes com doença cardíaca coronariana, analisar as trajetórias e taxas de detecção em cuidados de } \\
\text { saúde e avaliar a influência das doenças mentais e seu manejo na qualidade de vida e satisfação do paciente. De } \\
\text { resultado, obteve que } 33,8 \% \text { dos pacientes tinham doença mental e que } 49 \% \text { foram pré-diagnosticados corretamente. } \\
\text { Apenas } 30 \% \text { foram abordados pelos médicos para tratamento e só } 6,6 \% \text { realizavam psicoterapia e } 4,1 \% \text { terapia } \\
\text { medicamentosa. Como conclusão, os médicos precisam de mais treinamento para abordar adequadamente a } \\
\text { comorbidade mental. }\end{array}$ \\
\hline 12 & REAVELL J, et al. (2018) & $\begin{array}{l}\text { Consiste em uma metanálise que teve como objetivo avaliar a eficácia da Terapia Cognitivo-comportamental (TCC) } \\
\text { para depressão e ansiedade em pacientes com Doença Cardiovascular (DCV). Como resultado, teve que a depressão } \\
\text { (SMD - } 0,35, \text { IC 95\% -0,52 a -0,17) e ansiedade (SMD -0,34, IC 95\% -0,65 para -0,03) foram significativamente menores } \\
\text { em pacientes em TCC em comparação com o controle. De conclusão, teve que a TCC é uma alternativa eficaz para } \\
\text { reduzir ansiedade e depressão em pacientes com DCV e deve ser considerada no tratamento clínico padrão. }\end{array}$ \\
\hline 13 & TULLY PJ, et al. (2016) & $\begin{array}{l}\text { Essa revisão foca na ansiedade e seus subtipos em relação ao início e progressão da DCV, descrevendo diretrizes } \\
\text { úteis para identificar e tratar a ansiedade com eficácia. De ponto principal, tem-se que os transtornos de ansiedade } \\
\text { aumentam o risco de DCV incidente, mas sem demonstração de uma relação casual. Na conclusão, afirma que a } \\
\text { ansiedade indica prognóstico adverso nos pacientes com DCV, independente da depressão, e precisa de mais } \\
\text { intervenção clínica. }\end{array}$ \\
\hline 14 & WANG Y, et al. (2020) & $\begin{array}{l}\text { Trata-se de um estudo caso-controle que teve como objetivo investigar a associação entre os polimorfismos do gene } \\
\text { APLN/APLNR e o risco de depressão e ansiedade em pacientes com doença cardíaca coronária. Como resultado, } \\
\text { teve que os pacientes com a doença cardíaca que tinham um certo polimorfismo do alelo da apelina tinham um risco } \\
\text { maior de depressão e ansiedade. Assim, o artigo concluiu uma associação positiva de pacientes que tinham doença } \\
\text { cardíaca coronariana e esses polimorfismos com um maior risco de ter transtornos psiquiátricos. }\end{array}$ \\
\hline 15 & WESTERMAIR AL, et al. (2018) & $\begin{array}{l}\text { Esse estudo teve como objetivo trazer dados mais consistentes sobre as taxas de utilização dos cuidados de saúde } \\
\text { mental e a eficiência do tratamento em pacientes com Doença Arterial Coronariana (DAC). Como resultado, teve que } \\
\text { apenas } 21 \% \text { dos pacientes com DAC tiveram a aplicação da utilização global de cuidados de saúde mental desde o } \\
\text { início. Na conclusão, tiveram evidências de diagnóstico e tratamento insuficiente de transtorno de ansiedade em } \\
\text { pacientes com DAC, afirmando a necessidade de mais estudos prospectivos para corroborar ou falsificar os achados. }\end{array}$ \\
\hline
\end{tabular}

Fonte: Vasconcelos ST, et al., 2021. 


\section{DISCUSSÃO}

A ansiedade não diagnosticada pode causar preocupações para os cardiologistas devido as consultas recorrentes em consultórios e/ou unidades de emergências, além de sua apresentação clínica com sintomas cardíacos e respiratórios. Esses sintomas são reflexo da estimulação autonômica exacerbada e pode incluir a dor no peito, dispneia, palpitações, dormência, tonturas, aumento da frequência cardíaca e desconforto no Trato Gastrointestinal (TGI). Além desses, há também a manifestação no campo cognitivo, como o medo, a supressão de pensamentos e as preocupações em excesso e entre outros, bem como sintomas comportamentais, a exemplo as compulsões e a evasão de lugares ou situações (TULLY PJ, et al., 2016).

As inter-relações complexas entre os Transtornos de Ansiedade e o aumento do risco das DCVs não são inteiramente explicadas. Ainda há uma necessidade elucidativa para essa questão. Porém, existem alguns mecanismos fisiopatológicos que conseguem trazer uma explicação para essa associação. Essas emoções negativas decorrente da ansiedade simulam um estresse externo, causando uma desregulação dos circuitos neuro-hormonais. Esse desequilíbrio do controle do SNA causa um aumento do tônus simpático, elevando os níveis séricos de hormônios do estresse, como o cortisol e as citocinas pró-inflamatórias. Ainda, essas alterações sistêmicas podem induzir um estado pró-coagulante devido a uma exacerbação da atividade plaquetária e contribuindo para eventos ateroscleróticos (ANGERMANN CE e ERTL G, 2018).

Como uma das principais causas de DCV, a HAS tem uma prevalência em torno de $40 \%$ da população global. Os estudos que tentam explicar a causalidade entre a HAS e a ansiedade também não são tão elucidativos, mas sabe-se que há resultados significativos sobre uma associação positiva entre a ansiedade basal e taxas mais altas de desenvolver a HAS em um ano (OR 4,24; IC 95\% 1,29-14,01). Dentre todos os Transtornos de Ansiedade, notou-se que o TP (OR 1,2; IC 95\% 1,1-1,4) e a fobia social (OR 1,1; IC 95\% 1,0$1,3)$ são os tipos que mais se associam ao desenvolvimento de hipertensão, mesmo depois de ajustes para algumas variáveis. Além disso, pacientes que apresentavam mais de um distúrbio mental ou um diagnóstico mais cedo desses desarranjos eram mais propensos a desenvolver hipertensão (JOHNSON HM, 2019).

Já o IAM, outra patologia incluída no grupo das DCVs, é caracterizado por dispneia e dor torácica devido à isquemia e lesão do miocárdio, um músculo cardíaco. Ele pode ser dividido em Infarto Agudo do Miocárdio Com Supra do Segmento ST (IAMCSST) e Infarto Agudo do Miocárdio Sem Supra do Segmento ST (IAMSSST). Os Transtornos de Ansiedade são altamente prevalentes em pacientes com IAM, podendo apresentar os sintomas antes ou depois do episódio de infarto. A ansiedade preexistente é um preditivo independente para IAM, e a ansiedade após a lesão miocárdica causa uma piora do prognóstico do paciente (LI P, et al., 2020).

De acordo com um estudo feito, a incidência de sintomas de ansiedade em pacientes que sofreram Infarto Agudo do Miocárdio Sem Doença Arterial Coronariana Obstrutiva (IAMSDACO) foi de 30\%. Nesse trabalho, dos 620 participantes que foram selecionados, 186 tinham ansiedade. Nesse estudo, registrou 87 mortes e 151 eventos cardiovasculares adversos principais (morte cardiovascular, IC, acidente vascular cerebral e entre outros). Desses valores, houve 34 mortes e 56 eventos cardíacos no grupo com ansiedade. Todos esses valores correspondem a taxas não ajustadas de mortalidade por todas as causas, mas mesmo após esse ajuste, a ansiedade ainda permaneceu como um fator prognóstico independente para mortalidade por todas as causas, assim como os eventos cardiovasculares adversos principais (HE C, et al., 2020).

Ainda sobre o IAM, um outro estudo de coorte identificou que os pacientes que tinham ansiedade tendiam a ser mais jovens, do sexo feminino $(56,1 \%$ vs $36 \%)$, branco $(79,7 \%$ vs $70,1 \%)$ e maior incidência de comorbidades, como tabagismo $(29,4 \%$ vs $23 \%$ ) e hiperlipidemia ( $68 \%$ vs $64,7 \%)$. No entanto, curiosamente a incidência de diabetes (36,4\% vs $38,9 \%)$ e Doença Renal Crônica ( $21,6 \%$ vs $23,7 \%)$ foi menor no grupo com ansiedade. Ainda, no subgrupo que tinha IAMCSST apresentava 7,8\% dos pacientes com ansiedade e no subgrupo do IAMSSST, 10,9\% tinham ansiedade. Assim, percebe-se que a ansiedade tem efeitos diferentes sobre os subgrupos de IAM (LI P, et al., 2020).

A Doença Arterial Coronariana (DAC) é a forma mais comum de DCV e uma das principais causadoras de IAM. Atribui-se a maioria dos fatores de risco modificáveis, como os níveis de colesterol, hipertensão, 
diabetes, tabagismo, obesidade, dietas, álcool, fatores psicossociais e inatividade física. Embora muitos estudos associam a DAC com a depressão, há resultados sugestivos de que a ansiedade é um dos fatores de risco, mas as implicações e relações dessa associação não são bem compreendidas quanto às demais. De resultado, há números chamativos da influência da ansiedade na DAC, apresentando um aumento de 36$88 \%$ no risco de eventos cardíacos adversos. Ainda, salienta-se que a TAG e TP são de grande interesse e importância devido às taxas de prevalência ( $24 \%$ e $53 \%$ respectivamente) nessa doença cardíaca (FOLDESBUSQUE G, et al., 2020).

Esses transtornos mentais estão intimamente associados a menor qualidade de vida, maior mortalidade, maiores custos médicos e piores resultados somáticos (WESTERMAIR AL, et al., 2018). Um estudo mostrou que a ansiedade foi independentemente associada a um aumento do risco de mortalidade após eventos coronarianos agudos ((RR ajustado 1,21, IC 95\% 1,07-1,37, p=0,002), como a Síndrome Coronariana Aguda (SCA), uma das DACs. Os pacientes desse estudo que apresentavam ansiedade desde o início em conjunto com a SCA apresentavam um risco $21 \%$ maior de mortalidade e um risco de $47 \%$ maior para eventos cardíacos adversos maiores (morte cardíaca, IAM não fatal, reincidência de hospitalização cardíaca e entre outros). No entanto, vale ressaltar que a associação com a depressão pode ser um modificador significativo dessa associação (LI J, et al., 2020).

As razões que explicam a relação da DAC e os transtornos de ansiedade não são bem esclarecidas, mas há estudos afirmando um compartilhamento de mecanismos biológicos comuns entre esses desarranjos, como a inflamação, a disfunção endotelial e plaquetária e alterações do eixo hipotálamo-hipófise-adrenal. Ainda, há relatos de que a via de sinalização APLN / APLNR é uma candidata para explicar a associação entre DAC e os transtornos psicossomáticos, pois ela está envolvida na DCV, doença do Sistema Nervoso Central (SNC) e metabolismo energético. Ainda, os níveis aumentados de apelina sérica demonstraram ser preditivos no desenvolvimento de depressão e ansiedade em pacientes em diálise peritoneal, e os polimorfismos do seu gene está associado a um aumento de DAC (WANG Y, et al., 2020).

Devido à importância dos dados apresentados, percebe-se a importância de rastrear rotineiramente os transtornos mentais, especificamente os transtornos de ansiedade, em pacientes com DCV. Como essas doenças psicossomáticas atuam como uma barreira para adesão ao tratamento e relutância em mudar o estilo de vida, acarretando em uma redução da qualidade de vida do paciente, há fortes recomendações para o diagnóstico precoce e rastreamento de rotina. No entanto, infelizmente é improvável que os médicos façam um rastreio rotineiramente por causa das restrições da rotina diária, mesmo o diagnóstico sendo essencialmente clínico (PELTZER S, et al., 2020).

Dentre as várias diretrizes e manuais para diagnóstico dos transtornos mentais, podemos mencionar o Mini Exame do Estado Mental (MEEM) que examina memória de curto prazo, percepção visual, espacial e atenção, como um dos mais utilizados e de fácil aplicação. Além desse, há o "Patient Health Questionnaire9" (PHQ-9), recomendado pela American Heart Association como uma forma de triagem em pacientes com DCV. Nesse questionário, os pacientes respondem a frequência com que sentem os sintomas psiquiátricos, pontuando dependendo da resposta e classificando o grau da depressão de acordo com o escore obtido. Por fim, ainda há o "General Anxiety Disorder-7" (GAD-7) para diagnóstico de ansiedade e semelhante ao PHQ9, incluindo critérios mais relevantes para a TAG (ANGERMANN CE e ERTL G, 2018).

Os antidepressivos são terapias de escolha para a ansiedade, no entanto, ressalva-se que os tricíclicos não devem ser usados em pacientes com DCV devido a um aumento no risco de IAM. Diversas diretrizes recomendam o uso dos Inibidores Seletivos da Recaptação de Serotonina (ISRS), como o escitalopram e a sertralina, para o tratamento dos transtornos de ansiedade. Outros tratamentos, como os benzodiazepínicos, são outra escolha terapêutica, usada ainda mais em quadros de ansiedade flutuante. Ainda, como opções não medicamentosas consideram-se a Terapia Cognitivo-Comportamental (TCC) e os exercícios físicos (ANGERMANN CE e ERTL G, 2018; TULLY PJ, et al., 2016). Na TCC, os pacientes são ensinados a identificar pensamentos, monitorar o humor e controlar os comportamentos referente aos transtornos de ansiedade (REAVELL J, et al., 2018). 


\section{CONSIDERAÇÕES FINAIS}

A DCV como uma das patologias mais prevalentes, apresenta diversas etiologias, no entanto, os transtornos de ansiedade estão intrinsecamente associados ao aparecimento e pior prognóstico desta patologia, sendo considerados como um importante fator de risco para a DCV, embora necessite de mais estudos para explicar com fidedignidade a causa para essa questão. Com isso, devido à possibilidade direta de afetar a qualidade de vida do paciente, podendo ocasionar baixa adesão terapêutica e maior mortalidade, é necessário medidas e ações para rastreio e diagnóstico visando não trazer prejuízos para a vida do paciente e iniciando medidas preventivas e terapêuticas o mais rápido possível. Além disso, devido a algumas questões não esclarecidas com muito embasamento científico, como a associação da ansiedade na DCA, é necessário que mais estudos sejam feitos para elucidar essas relações. Assim, haverá uma oferta maior para a saúde da população, contribuindo para a qualidade de vida dos pacientes acometidos por esse quadro clínico.

\section{REFERÊNCIAS}

1. ANGERMANN CE, ERTL G. Depression, Anxiety, and Cognitive Impairment: Comorbid Mental Health Disorders in Heart Failure. Curr Heart Fail Rep., 2018; 15(6): 398-410.

2. CELANO CM, et al. Depression and Anxiety in Heart Failure: a Review. Harvard review of psychiatry, 2018; 26(4): 175-184.

3. FOLDES-BUSQUE G, et al. Epidemiology and prognostic implications of panic disorder and generalized anxiety disorder in patients with coronary artery disease: rationale and design for a longitudinal cohort study. BMC Cardiovascular Disorders, 2020; $21(26): 1$.

4. FOLDES-BUSQUE G, et al. Epidemiology and prognostic implications of panic disorder and generalized anxiety disorder in patients with coronary artery disease: rationale and design for a longitudinal cohort study. BMC Cardiovascular Disorders, 2020; 21(26): 1.

5. GARCÍA-BATISTA ZE, et al. Depresión, ansiedad e ira en adultos que sufrieron ataque al miocardio: un estudio de caso-control. Revista de Psicología, 2020; 38(1): 1.

6. HE C, et al. Association between anxiety and clinical outcomes in Chinese patients with myocardial infarction in the absence of obstructive coronary artery disease. Clinical Cardiology, 2020; 43(7): 659-665.

7. JOHNSON HM. Anxiety and Hypertension: Is There a Link? A Literature Review of the Comorbidity Relationship Between Anxiety and Hypertension. Curr Hypertens Rep. 2019; 21(9): 66.

8. KANG H, et al. Impact of Anxiety and Depression on Physical Health Condition and Disability in an Elderly Korean Population. Psychiatry Investigation, 2017; 14(3): 240-248.

9. LI J, et al. Anxiety and clinical outcomes of patients with acute coronary syndrome: a meta-analysis. BMJ OPEN, 2020; $1(1): 1$.

10. LI P, et al. The association between anxiety disorders and in-hospital outcomes in patients with myocardial infarction. Clinical Cardiology, 2020; 43(6): 622-629.

11. MACHADO MB, et al. Prevalência de transtornos ansiosos e algumas comorbidades em idosos: um estudo de base populacional. J. bras. psiquiatr., 2016; 65(1): 1.

12. PELTZER S, et al. Detection and treatment of mental disorders in patients with coronary heart disease (MenDis-CHD): A cross-sectional study. PLOS ONE, 2020; 15(12): 1.

13. REAVELL J, et al. Effectiveness of Cognitive Behavioral Therapy for Depression and Anxiety in Patients With Cardiovascular Disease: A Systematic Review and Meta-Analysis. Psychosom Med., 2018; 80(8): 1.

14. TULLY PJ, et al. Anxiety and Cardiovascular Disease Risk: a Review. Curr Cardiol Rep., 2016; 18(12): 1.

15. WANG Y, et al. Association of Apelin and Apelin Receptor Polymorphisms With the Risk of Comorbid Depression and Anxiety in Coronary Heart Disease Patients. Frontiers in Genetics, 2020; 1(1): 1.

16. WESTERMAIR AL, et al. Utilization of Mental Health Care, Treatment Patterns, and Course of Psychosocial Functioning in Northern German Coronary Artery Disease Patients with Depressive and/or Anxiety Disorders. Frontiers in Psychiatry, 2018; 9(75): 1. 